\title{
Impurity scattering in the bulk of topological insulators
}

\author{
Cheung Chan and Tai-Kai $\mathrm{Ng}$ \\ Department of Physics, The Hong Kong University of Science and Technology, Clear Water Bay, Hong Kong
}

(Received 7 November 2011; revised manuscript received 28 February 2012; published 26 March 2012)

\begin{abstract}
We study in this paper time-reversal $\delta$-impurity scattering effects in the bulk of topological insulators (TI) in two and three dimensions. Specifically we consider how impurity scattering strength is affected by the bulk band structure of topological insulators. An interesting band inversion effect associated with the change of the system from ordinary to topological insulator is pointed out. Experimental consequences of our findings are discussed.
\end{abstract}

DOI: 10.1103/PhysRevB.85.115207

PACS number(s): 71.23.An, 73.20.Hb, 61.72.J-

\section{INTRODUCTION}

Topological insulators (TI) are insulators characterized by a $\mathbb{Z}_{2}$ topological number of the bulk band structure, and realize a new topological quantum state protected by time-reversal symmetry. ${ }^{1,2}$ Theoretically, TI are insulating in the bulk but possess gapless boundary states of helical Dirac fermions that give rise to interesting transport properties not realized in ordinary insulators (OI). Novel excitations like Majorana zero modes ${ }^{3}$ have been proposed to exist in this class of materials and applications of the materials in spintronics have also been discussed. ${ }^{4}$

Toward the applications of TI, a better understanding of the overall properties of the materials beyond their topological surface excitations is needed. For example, to utilize exotic boundary states of TI one needs to ensure a sufficient insulating bulk resistivity, which is a challenge at present due to the presence of bulk conductivity ${ }^{5-10}$ largely arising from the presence of impurities and defects in the materials. ${ }^{11}$ If the defects are external, which is the case for doped materials, one way to attain high bulk resistivity is to synthesize the materials with delicate balance between donors and acceptors. ${ }^{12,13}$ On the other hand, defects can also affect the bulk conductivity through impurity induced in-gap bound states that lead to impurity band and effective narrowing of band gap.

It was pointed out in Ref. 3 that point-like defects in TI do not give rise to topological protected zero-energy states. However, in-gap bound states can still be induced by (isolated) impurities ${ }^{14-16}$ through conventional mechanisms. For example, depletion of wave function at the defect results ingap bound states if one imagines that the impurity is formed by bending and shrinking the edge into a localized defect. ${ }^{14}$ However, unlike the (real) edge states, these localized bound states are finite energy modes because they suffer from symmetry allowed (self) interactions. In this paper we consider isolated time-reversal impurities described by $\delta$-function potentials, and study how impurity scattering can depend on the special band structure of TI. We shall show that impurity scattering can be enhanced in the bulk TI compared with OI due to particular band structure associated with the materials. A criteria to search for materials with suppressed impurity scattering effect is given. An interesting sign-inversion effect in impurity scattering associated with the band inversion in TI-OI transition is also pointed out. Experimental implications of these effects are discussed.

\section{FORMULATION}

We adopt the modified Dirac Hamiltonian in either two or three dimensions (2D or 3D) as effective models for the topological insulator, ${ }^{14-17}$ that is,

$$
H=\sum_{\mathbf{k}, s} \psi_{\mathbf{k} s}^{\dagger} h_{2(3) \mathrm{D}}(\mathbf{k}, s) \psi_{\mathbf{k} s},
$$

where $\psi_{\mathbf{k} s}^{\dagger}\left(\psi_{\mathbf{k} s}\right)$ are two-component Dirac fields with momentum and spin indices $\mathbf{k}$ and $s=\uparrow, \downarrow$, respectively, and

$$
h_{2 \mathrm{D}}(\mathbf{k}, s)=k_{x} \sigma^{y}+k_{y} \sigma^{x} s^{z}+m_{k} \sigma^{z}
$$

for 2D topological insulators where $\sigma$ and $s$ are Pauli matrices, and $m_{k}=m-B k^{2} \cdot \sigma^{z}=+1(-1)$ describes two contributing atomic orbitals to the topological insulator. For example, they represent $s$ and $p$ states for the $\mathrm{HgTe} / \mathrm{CdTe}$ system. The model can also be generalized to describe 3D topological insulators with a similar effective Hamiltonian $h_{3 \mathrm{D}}(\mathbf{k}, s)$ with $m_{k}=m-$ $\sum_{i} B_{i} k_{i}^{2}$, where $i=x, y, z$ and $B_{i}$ are parameters of the same sign. We shall for simplicity consider $B_{x}=B_{y}=B \neq B_{z}$, corresponding to a common type of topological insulators in 3D with crystal structure $R 3 \mathrm{~m}$.

For $m B_{i}<0$, the bands are ordered conventionally throughout the Brillouin zone and the system is a ordinary (topologically trivial) insulator. The bands near $\mathbf{k}=0$ are inverted due to strong spin-orbit coupling for $m B_{i}>0$ and the system becomes a topological insulator. The energy eigenvalues of the bulk Hamiltonians are given by $\omega_{k}=$ $\pm \sqrt{k^{2}+\left(m-\sum B_{i} k_{i}^{2}\right)^{2}}$ and are doubly degenerate (Kramers degeneracy). The time reversal operator is given by $\hat{\mathcal{T}}=i s^{y} \hat{K}$ with $\hat{\mathcal{T}}^{2}=-1$, where $\hat{K}$ is the complex conjugate operator.

In real materials there are other electronic bands and impurity scatterings exist in general between the Dirac bands localized near the $\Gamma$ point and the other electronic bands. We expect that scattering effects between Dirac bands and the other bands will be strongest near band extrema. To capture these effects we consider also interband scattering between the Dirac bands with a model quadratic band described by

$$
H_{\mathrm{q}}=\sum_{\mathbf{k} s}\left(\frac{k^{2}}{2 M}+\mu\right) c_{\mathbf{k} s}^{\dagger} c_{\mathbf{k} s}
$$

where $M$ and $\mu$ are chosen to have the same sign so that the quadratic band does not cross the Fermi level and the system remains a bulk insulator. $M, \mu>0(<0)$ refers to 
a conduction (valence) band in this notation. We note that these band extrema are not necessarily far away from the Fermi level, hinted by first-principle calculations. ${ }^{18}$ These low lying extrema may also contribute significantly to the impurity scatterings.

We shall describe the impurity scattering by a single $s$-wave $\delta$-function potential sitting at the origin in this paper. In the four-band modified Dirac model we adopt here, the impurity scattering term within the topological bands can be written in orbital basis ${ }^{19}$ as

$$
H_{I 1}=\hat{u} \delta(\vec{x}),
$$

where $\hat{u}$ is a $4 \times 4$ matrix. For time reversal impurities $\hat{u}$ should be Hermitian and time reversal symmetric, that is, $\hat{\mathcal{T}} \hat{u} \hat{\mathcal{T}}^{-1}=$ $\hat{u}$. There are six bases for $\hat{u}$, denoted as $u^{(i=0, \ldots, 5)}=\left(I, \sigma^{x}, \sigma^{z}\right.$, $\left.\sigma^{y} s^{x}, \sigma^{y} s^{y}, \sigma^{y} s^{z}\right)$ and $\hat{u}=\sum_{i} \alpha_{i} u^{(i)}$ in general, where $\alpha_{i}$ are parameters characterizing the impurity potential.

To describe scattering between the Dirac and quadratic bands, we consider

$$
H_{I 2}=\sum_{\sigma, s} v_{\sigma}\left[\Psi_{s}^{\dagger}(0) \psi_{\sigma s}(0)+\psi_{\sigma s}(0)^{\dagger} \Psi_{s}(0)\right],
$$

where $\sigma= \pm 1$ and $s=\uparrow, \downarrow$. $\Psi_{s}(x)$ is the Fourier transform of $c_{\mathbf{k} s}$. We include only spin-independent scattering in writing down $H_{I 2}$.

The effect of impurity scattering is described by the $T$ matrix defined by

$$
\hat{T}(\omega)=\left[\mathbb{I}-\hat{U} \hat{G}_{0}(\omega)\right]^{-1} \hat{U}
$$

for the $s$-wave $\delta$-function potential, where $\hat{U}$ is a $6 \times 6$ impurity scattering matrix

$$
\hat{U}=\left[\begin{array}{ll}
\hat{u} & \hat{v} \\
\hat{v}^{\dagger} & \hat{w}
\end{array}\right]
$$

where $\hat{u}$ describes the impurity scattering within the Dirac bands, $\hat{w}$ is a diagonal $2 \times 2$ matrix describing impurity scattering within the quadratic band, and $\hat{v}$ is a $4 \times 2$ matrix describing scattering between Dirac and quadratic bands [Eq. (5)]. $\hat{G}_{0}(\omega)=\sum_{\mathbf{k}} \hat{G}_{0}(\mathbf{k}, \omega)$ is the corresponding $6 \times 6$ on-site matrix Green's function.

With the modified Dirac and quadratic bands, $\hat{G}_{0}$ and $\hat{U}$ are explicitly given by

$$
\hat{G}_{0}(\omega)=\left[\begin{array}{cc}
\hat{G}_{0}^{\mathrm{Dirac}}(\omega) & 0 \\
0 & \hat{G}_{0}^{\mathrm{q}}(\omega)
\end{array}\right],
$$

where

$$
\hat{G}_{0}^{\mathrm{q}}(\omega)=\left[\begin{array}{cc}
g_{0}^{\mathrm{q}}(\omega) & 0 \\
0 & g_{0}^{\mathrm{q}}(\omega)
\end{array}\right]
$$

is the on-site Green's function of the quadratic band in the spin $s^{z}$ basis [see Eq. (14)].

For the modified Dirac model, the on-site matrix Green's function in the basis $[(1,1),(-1,1),(1,-1),(-1,-1)]$ [with respect to $\left.\left(\sigma^{z}, s^{z}\right)\right]$ is given by

$$
\hat{G}_{0}^{\text {Dirac }}(\omega)=\left[\begin{array}{llll}
g^{+}(\omega) & & & \\
& g^{-}(\omega) & & \\
& & g^{+}(\omega) & \\
& & & g^{-}(\omega)
\end{array}\right] \text {, }
$$

where

$$
g^{ \pm}(\omega)=-\int d \Omega_{D} \int_{0}^{\Lambda} k^{D-1} d k \frac{\omega \pm m_{k}}{k^{2}+m_{k}^{2}-\omega^{2}},
$$

where $\Omega_{D}$ is the solid angle in dimension $D=2,3 . \Lambda$ is a high energy cutoff above which the effective Dirac model (2) is inadequate in describing the band structure. Notice that the on-site Green's function matrix $\hat{G}_{0}$ is diagonal. This is a special feature of the $\delta$-function impurity potential and would be absent if the impurity potential includes higher angular momentum components. In particular, the existence of impurity bound state is determined by the eigenvalue equation $^{20,21}$

$$
\operatorname{det}\left[\mathbb{I}-\hat{G}_{0}(\omega) \hat{U}\right]=0
$$

\section{III. $g^{ \pm}(\omega)$ AROUND BAND EDGE AND ENHANCED IMPURITY SCATTERING}

Without loss of generality, we shall assume $B>0$ in the following. We note that in the limit of weak impurity scattering, impurity-induced in-gap bound states can exist only if $g^{ \pm}(\omega)$ diverges near the band edge. In usual (2D) semiconductors the divergence in $g^{ \pm}(\omega)$ is originated from the band extremum associated with a quadratic band structure. In TI the band structure is more complicated and the band extremum may locate at a line of $\vec{k}$ values leading to stronger divergence behavior in $g^{ \pm}(\omega)$. This phenomenon may arise in both 2D and 3D TI and will lead to enhanced impurity scattering compared with usual semiconductors. First we consider 2D.

\section{A. $2 m B<1$}

The integral (11) for $g^{ \pm}(\omega)$ can be evaluated exactly in $2 \mathrm{D}$. For $2 m B<1$ the band minimum is located at $k=0$ with band gap $|m|$ as in usual semiconductors. It is straightforward to show that in this case

$$
\left.g^{ \pm}(\omega)\right|_{|\omega| \rightarrow|m|^{-}} \rightarrow-\pi(\omega \pm m) \ln \left(1+\frac{\delta^{2}}{m^{2}-\omega^{2}}\right),
$$

where $\delta \sim \min \left(\Lambda, \sqrt{\left|\frac{m}{B}\right|}\right)$ is the momentum cutoff below which we may approximate $m_{k} \sim m$. We note that a logarithmic divergence appears in $g^{ \pm}(\omega)$ near the band edge when $|\omega| \rightarrow$ $\mathrm{m}^{-}$. Similar logarithmic divergence occurs for quadratic bands with Green's function given by

$$
g_{0}^{\mathrm{q}}(\omega)=-2 \pi M \ln \left[1+\frac{\Lambda^{2}}{2 M(\mu-\omega)}\right] .
$$

The logarithmic divergence arises from the quadratic dispersion of electron dispersion near the band edge and is responsible for appearance of impurity bound state for arbitrarily weak attractive scattering potential in 2D. Taking $B>0$, we find that $g^{+(-)}(\omega)$ has logarithmic divergence at $\omega \rightarrow+(-)|m|$ to $-(+) \infty$ for topological insulators (TI) $(m>0)$ and has logarithmic divergence at $\omega \rightarrow-(+)|m|$ to $+(-) \infty$ for ordinary insulators $(\mathrm{OI})(m<0)$, implying that the role of $g^{+(-)}(\omega)$ is inverted when the system changes from OI to TI. Microscopically, a system changes from OI to $\mathrm{TI}$ when there is an orbital inversion $(2 m=$ difference in 
energy between the two orbitals forming the inverted bands) and the inversion in the role of $g^{ \pm}(\omega)$ is a direct consequence of this orbital inversion. In other words, there is a direct correspondence between $g_{\mathrm{TI}}^{ \pm}(\omega) \leftrightarrow g_{\mathrm{OI}}^{\mp}(\omega)$ with the same band gap $|m|$.

\section{B. $2 m B>1$}

In this case, the band extremum occurs at a line of momentum $\vec{k}$ with $|\vec{k}|=k_{0}$. The corresponding band gap is given by $\Delta=\frac{\sqrt{4 m B-1}}{2|B|}$. Evaluating the integral, we find

$$
\left.g^{ \pm}(\omega)\right|_{|\omega| \rightarrow \Delta^{-}} \rightarrow-\frac{\pi^{2}}{\sqrt{2|B|}} \frac{\omega \pm \frac{1}{2 B}}{\sqrt{\Delta^{2}-\omega^{2}}}+\text { const. }
$$

We see that $g^{ \pm}(\omega)$ has a stronger (inverse square-root) divergence at the gap edge compared with the case $2 m B<1$. This is a result of "dimension reduction" in the integral when the band minimum occurs at a ring of momentum $\vec{k}$ with $|\vec{k}|=k_{0}$. We notice that $g^{+}(\omega)$ diverges to $-(+) \infty$ at $\omega \rightarrow+(-) \Delta$, with a weaker divergence (weighting factor) at $\omega \rightarrow-\Delta$. A similar situation occurs for $g^{-}(\omega)$ which diverges to $+(-) \infty$ at $\omega \rightarrow-(+) \Delta$, with a weaker divergence at $\omega \rightarrow \Delta$. The appearance of divergences at both $\omega= \pm \Delta$ is possible because of hybridization between the two atomic orbitals. We note that similar double-divergent behavior cannot occur for ordinary insulators which exist only at $m B<0$.

\section{3D case}

We can study $g^{ \pm}(\omega)$ for 3D TI in a similar way. Using the Hamiltonian $h_{3 \mathrm{D}}(\vec{k}, s)$ with $B_{x}=B_{y}=B \neq B_{z}$ we find that the divergent behavior of $g^{ \pm}(\omega)$ can be classified in three different types as shown in Fig. 1. Type $I$ is defined by $2 m\left(B_{z}, B\right)<1$ with band extremum occurring at $\vec{k}=0$. Type II is defined by $B>\max \left(\frac{1}{2 m}, B_{z}\right)$ with band extremum occurring at a ring of $\vec{k}$ values, $\vec{k}=\left(k_{x}, k_{y}, 0\right)$ with $k_{x}^{2}+k_{y}^{2}=$ $k_{0}^{2}$ where $k_{0}=\frac{1}{B} \sqrt{m B-\frac{1}{2}}$. Type III is defined by $B_{z}>$ $\max \left(\frac{1}{2 m}, B\right)$ with band extrema occurring at $\vec{k}=\left(0,0, \pm k_{0}\right)$, where $k_{0}=\frac{1}{B_{z}} \sqrt{m B_{z}-\frac{1}{2}}$.

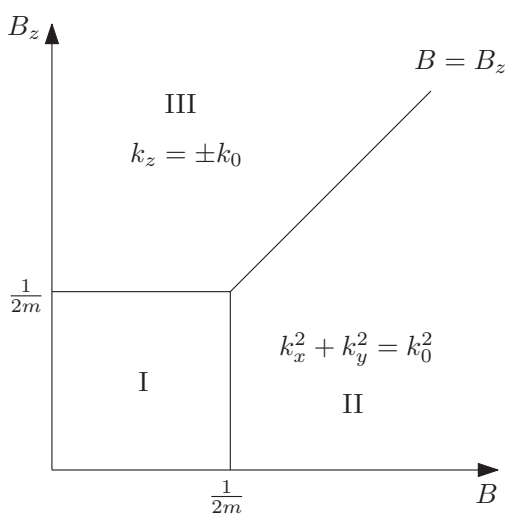

FIG. 1. Parameter range for band extrema $k_{0}$.
The corresponding Green's functions are given by

$$
\begin{aligned}
\frac{g_{\mathrm{I}}^{ \pm}(\omega)}{-4 \pi} & \sim \int_{0}^{\delta} k^{2} d k \frac{\omega \pm m}{k^{2}+m^{2}-\omega^{2}} \\
& =(\omega \pm m)\left[\delta-\sqrt{m^{2}-\omega^{2}} \tan ^{-1}\left(\frac{\delta}{\sqrt{m^{2}-\omega^{2}}}\right)\right] \\
& \sim \text { const. as }|\omega| \rightarrow|m|^{-}, \\
\frac{g_{\mathrm{II}}^{ \pm}(\omega)}{-2 \pi} & \sim \int_{k_{0}-\frac{\delta}{2}}^{k_{0}+\frac{\delta}{2}} d k \int_{0}^{\delta_{z}} d k_{z} \frac{k_{0}\left[\omega \pm\left(m-B k_{0}^{2}\right)\right]}{k^{2}+k_{z}^{2}+m_{k}^{2}-\omega^{2}} \\
& \sim \frac{\omega \pm \frac{1}{2 B}}{\sqrt{B\left(B-B_{z}\right)}} \ln \left(\frac{1}{\sqrt{\Delta^{2}-\omega^{2}}}\right) \text { as }|\omega| \rightarrow \Delta^{-},
\end{aligned}
$$

$$
\begin{aligned}
\frac{g_{\mathrm{III}}^{ \pm}(\omega)}{-2 \pi} & \sim \sum_{\gamma= \pm} \int_{0}^{\delta} d k \int_{\gamma k_{0}-\frac{\delta_{z}}{2}}^{\gamma k_{0}+\frac{\delta_{z}}{2}} d k_{z} \frac{k\left[\omega \pm\left(m-B_{z} k_{0}^{2}\right)\right]}{k^{2}+k_{z}^{2}+m_{k}^{2}-\omega^{2}} \\
& \sim \text { const. as }|\omega| \rightarrow \Delta^{-}
\end{aligned}
$$

where $\Delta=\frac{1}{2|\bar{B}|} \sqrt{4 m \bar{B}-1}$, where $\bar{B}=B\left(B_{z}\right)$ of type II(III). Here $\delta, \delta_{z}$ are momentum cutoff as in Eq. (13). We notice that logarithmic divergence in $g^{ \pm}(\omega \rightarrow \pm \Delta)$ is found for TI of type II which is absent in usual semiconductors which exist only at $m \bar{B}<0$. The sign-inversion effect in $g_{\mathrm{I}}^{ \pm}$is also clear although there is no divergence compared with its $2 \mathrm{D}$ counterpart.

\section{Enhanced impurity scattering}

The divergence behavior of $g^{ \pm}(\omega)$ has strong implications on impurity scattering behavior described by the $T$ matrix (6) since impurity scattering can be strongly enhanced if $g^{ \pm}(\omega)$ diverges. In particular TI with nondiverging $g^{ \pm}(\omega)$ are more robust toward impurity scattering and are better candidates for transport application.

Thus (3D) materials belonging to type I and III are the preferred materials for transport application. For type II, due to the "dimension reduction" effect, divergence appears near the band edges at both $\omega \rightarrow \pm \Delta$ and impurity scattering effects are much enhanced compared with usual semiconductors. Notice that materials where all $B$ are not equal do not suffer from dimension reduction effect and are "preferred" also from the point of view of impurity scattering.

We now apply our analysis to the three TI materials given in Ref. 22, where the low energy spectra around the inverted bands are given by

$$
\omega_{k}=\epsilon_{k} \pm \sqrt{A_{0}^{2} k_{\|}^{2}+B_{0}^{2} k_{z}^{2}+M_{k}^{2}}
$$

where $\epsilon_{k}=C_{1} k_{z}^{2}+C_{2} k_{\|}^{2}, \quad M_{k}=M_{0}+M_{1} k_{z}^{2}+M_{2} k_{\|}^{2}$, and $k_{\|}^{2}=k_{x}^{2}+k_{y}^{2}$. Here we neglect the higher order term $H_{3}$ which is relevant only for large $k$. Using the fitting parameters given in Ref. 22 we classify both the upper and lower topological bands for the three materials in Table I. We see from Table I that $\mathrm{Bi}_{2} \mathrm{Se}_{3}$ and $\mathrm{Bi}_{2} \mathrm{Te}_{3}$ both have type II band structure and $\mathrm{Sb}_{2} \mathrm{Te}_{3}$ is the preferred material among the three as far as transport application is concerned. 
TABLE I. Band extrema $k_{0}$ and corresponding types in Fig. 1 of the inverted band for $\mathrm{Bi}_{2} \mathrm{Se}_{3}, \mathrm{Bi}_{2} \mathrm{Te}_{3}$, and $\mathrm{Sb}_{2} \mathrm{Te}_{3}$ (band parameters are coming from Ref. 22).

\begin{tabular}{|c|c|c|c|c|c|c|}
\hline \multirow{2}{*}{$\begin{array}{l}\text { Material } \\
\text { band }\end{array}$} & \multicolumn{2}{|c|}{$\mathrm{Bi}_{2} \mathrm{Se}_{3}$} & \multicolumn{2}{|c|}{$\mathrm{Bi}_{2} \mathrm{Te}_{3}$} & \multicolumn{2}{|c|}{$\mathrm{Sb}_{2} \mathrm{Te}_{3}$} \\
\hline & upper & lower & upper & lower & upper & lower \\
\hline$k_{0} / \AA^{-1}$ & 0 & 0.09 & 0 & 0.04 & 0.11 & 0.08 \\
\hline Type & I & II & I & II & III & III \\
\hline
\end{tabular}

\section{IMPURITY-INDUCED IN-GAP BOUND STATES}

In this section we consider the formation of impurity induced bound states in the bulk where we shall illustrate the existence of an interesting band-inversion effect. First we focus on the intra-Dirac bands scattering. The scattering matrix respecting $\mathcal{T}$ can be written in orbital basis [the same basis as used in writing down Eq. (10)] as

$$
\hat{u}=\left[\begin{array}{cccc}
u_{11} & u_{I} & 0 & \tilde{u}_{I} \\
u_{I}^{*} & u_{22} & -\tilde{u}_{I} & 0 \\
0 & -\tilde{u}_{I}^{*} & u_{11} & u_{I}^{*} \\
\tilde{u}_{I}^{*} & 0 & u_{I} & u_{22}
\end{array}\right],
$$

where $u_{11,22} \in \mathbb{R}$ and $u_{I}, \tilde{u}_{I} \in \mathbb{C}$.

\section{A. Single channel scattering study}

To see the qualitative effect of band structure and TI-OI inversion on bound state formation we consider the case of single scattering channels, that is, when only one of the $u$ in Eq. (20) is nonzero. In this case it is straightforward to obtain the following eigenvalue equations:

$$
\begin{gathered}
{\left[1-u_{11} g^{+}(E)\right]^{2}=0,} \\
{\left[1-u_{22} g^{-}(E)\right]^{2}=0,} \\
{\left[1-\left|u_{I}\right|^{2} g^{+}(E) g^{-}(E)\right]^{2}=0,} \\
{\left[1-\left|\tilde{u}_{I}\right|^{2} g^{+}(E) g^{-}(E)\right]^{2}=0}
\end{gathered}
$$

for each nonzero $u$ where $E$ is the bound state energy. We shall analyze in detail the above equations for the case $2 m B<1$ in $2 \mathrm{D}$. The analysis can be easily generalized to the case $2 m B>$ 1 and to three dimensions as we shall see in the following.

We start with inter-Dirac-band scattering. Since $g^{+}(E) g^{-}(E)<0$ [cf. Eq. (13)] in the gap region $|E|<|m|$, the scattering matrix $T$ has no divergence and there is no bound state induced by inter-Dirac-band scattering. The situation is very different for intraband scattering where existence of in-gap bound state depends on the sign of $u_{i i} g^{ \pm}(E)$, and it is easy to see that a bound state which appears in the conduction/valence band in OI $(m<0)$ state disappears in the corresponding TI $(m>0)$ state and vice versa, where we have again fixed $B>0$ for brevity. The results of bound state formation is summarized in Table II. The doubly degeneracy of bound state solutions is a result of Kramer's degeneracy coming from time reversal symmetry. The existence of bound states in these cases is a natural result of an attractive (repulsive) impurity in electron (hole) liquid, which is known to induce bound state in two dimensions for arbitrarily weak
TABLE II. Single channel intra-Dirac bands scattering induces ingap bound state in the weak scattering limit. $m$ is the "gap" parameter in the modified-Dirac Hamiltonian.

\begin{tabular}{lcc}
\hline \hline$u_{11} \neq 0$ [Eq. (21)] & $u_{11}>0$ & $u_{11}<0$ \\
\hline$m>0$ & No & Yes \\
$m<0$ & Yes & No \\
$u_{22} \neq 0$ [Eq. (22)] & $u_{22}>0$ & $u_{22}<0$ \\
\hline$m>0$ & Yes & No \\
$m<0$ & No & Yes \\
\hline \hline
\end{tabular}

potential. The sign change in $m$ just reverses the conduction band to valence band or vice versa but the signs of $u$ are not reversed, resulting in the appearance/disappearance of impurity bound states when $m$ changes sign.

We next consider impurity-induced interband scattering between the Dirac bands and an extra quadratic band described by Eq. (5). For simplicity we again consider single scattering channel with either $v_{+(-)}$being nonzero. It is straightforward to obtain the eigenvalue equations

$$
\begin{aligned}
& {\left[1-v_{+}^{2} g^{+}(E) g_{0}^{\mathrm{q}}(E)\right]^{2}=0,} \\
& {\left[1-v_{-}^{2} g^{-}(E) g_{0}^{\mathrm{q}}(E)\right]^{2}=0,}
\end{aligned}
$$

where we again notice the Kramer's degeneracy of the solutions. Notice that unlike inter-Dirac-bands scattering where $g^{+}(\omega) g^{-}(\omega)<0$, independent of the sign of $m, g^{ \pm}(E) g_{0}^{\mathrm{q}}(E)$ depends now on the sign of $m$. The resulting bound state formation possibilities are summarized in Table III where we have considered the quadratic band to be either a conduction or valence band. Notice that the results are independent of the sign of the impurity potential since $v_{\sigma}$ always enters the eigenvalue equation as $v_{\sigma}^{2}$. Again if we fix the scattering channel and the quadratic band, we find that the bound state appears/disappears when $m$ changes sign because the sign of $g^{+(-)}(E)$ changes upon orbital inversion.

Naively, we expect that impurity-induced intra-Dirac bands scattering will be more important than inter-Dirac-quadratic bands scattering because the Dirac and quadratic bands are located at different parts of the Brillouin zone, and the interDirac-quadratic band impurity scattering matrix element $v$ will in general be weaker in magnitude than the intra-Dirac bands scattering matrix element $u$. Our analysis suggests that because of the nontrivial signs associated with the Green's functions $g^{ \pm}$ and $g_{0}^{\mathrm{q}}$, this is not always true and it is possible that impurity

TABLE III. Induced in-gap bound states resulting from weak inter-Dirac-quadratic band scatterings. Quadratic bands are described by $M, \mu>0$ (conduction) or $<0$ (valence), and $m$ is the gap parameter of the modified-Dirac equation.

\begin{tabular}{lcc}
\hline \hline$v_{+} \neq 0$ [Eq. (25)] & $M, \mu>0$ & $M, \mu<0$ \\
\hline$m>0$ & Yes & No \\
$m<0$ & No & Yes \\
$v_{-} \neq 0$ [Eq. (26)] & $M, \mu>0$ & $M, \mu<0$ \\
\hline$m>0$ & No & Yes \\
$m<0$ & Yes & No \\
\hline \hline
\end{tabular}


induced bound states may form from inter-Dirac-quadratic band scattering but not from intra-Dirac band scattering.

Our analysis can be extended easily to the case $2 m B>1$. There is no band-inversion effect in this case since ordinary insulator exists only for $m B<0$ and impurity bound state always exist because of the "double-divergence" behavior in $g^{ \pm}(\omega)$. The situation for 3D TI are similar. Band inversion effect exist only in type I and weak impurity bound state always exist in type II.

\section{B. Numerical study}

To further investigate the impurity effect with multiple scattering channels and finite scattering strength, we consider a system described by 3D modified Dirac bands with [cf. Eq. (20)]

$$
\hat{u}=\left[\begin{array}{cccc}
u_{11} & u_{I} & 0 & 0 \\
u_{I}^{*} & u_{22} & 0 & 0 \\
0 & 0 & u_{11} & u_{I}^{*} \\
0 & 0 & u_{I} & u_{22}
\end{array}\right],
$$

where we only consider the scattering channels without spin flip. The eigenvalue equation is given by

$$
\begin{aligned}
0 & =\operatorname{det}\left[\mathbb{I}-\hat{G}_{0}^{\text {Dirac }}(E) \hat{u}\right] \\
& =\left[\left(u_{11} u_{22}-\left|u_{I}\right|^{2}\right) g^{+} g^{-}-\left(u_{11} g^{+}+u_{22} g^{-}\right)+1\right]^{2},
\end{aligned}
$$

which is then solved numerically and the results are shown in Fig. 2.
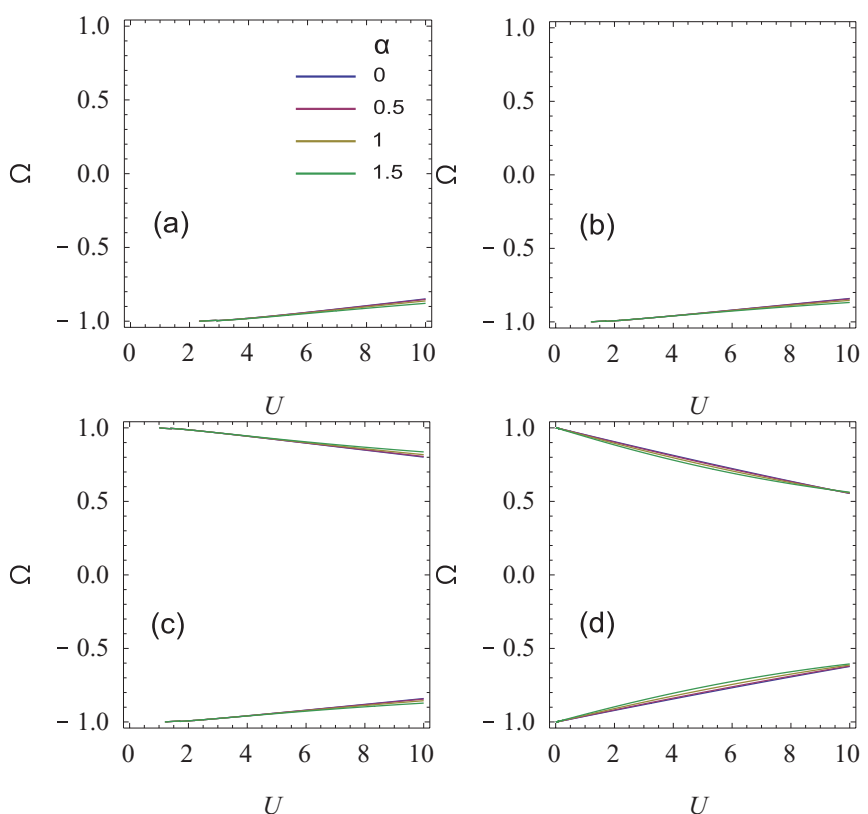

FIG. 2. (Color online) In-gap bound state spectrum, where $\Omega=$ $\frac{\omega}{\Delta}$ is the energy $\omega$ normalized with the gap $\Delta$ and here $\left|u_{I}\right|=\alpha U$ with $\stackrel{\Delta}{\alpha}=0,0.5,1,1.5$. (a) Ordinary insulator (OI) with $\frac{1}{U}\left(u_{11}, u_{22}\right)=$ $(1.2,1),\left(m, B, B_{z}\right)=(-0.2,1,1)$. (b) TI of type I (TI-I) (cf. Fig. 1) with parameter similar to (a) except $m=0.2$. (c) TI-I similar to (b) but sign of $u_{11}$ changed, that is $\frac{1}{U}\left(u_{11}, u_{22}\right)=(-1.2,1)$. OI with $m=-0.2$ would give no in-gap solutions. (d) TI of type II (TI-II) with $\frac{1}{U}\left(u_{11}, u_{22}\right)=(-1.2,1),\left(m, B, B_{z}\right)=(1,1,0.5)$. In all subfigures we have $\delta=\delta_{z}=0.1$.
We first consider the sign inversion effect in the presence of multiple scattering channels. Figures 2(a) and 2(b) show the in-gap bound state spectrum with $\operatorname{sgn}\left(u_{11}\right)=\operatorname{sgn}\left(u_{22}\right)>0$ in an OI and an TI-I, respectively. That the spectra look alike is consistent with the results in Table II. Since the signs of $u_{11}$ and $u_{22}$ are the same and positive, bound states exist near the valence band edge, even if band inversion occurs (i.e., $m$ changes sign). However, given the degree of freedom in the scattering matrix [Eq. (27)], we can choose the relative sign of $u_{11}$ and $u_{22}$ such that bound states only exist in either OI or TI-I (cf. Table II). Figure 2(c) shows the spectrum in TI-I with the choice $\operatorname{sgn}\left(u_{22}\right)=-\operatorname{sgn}\left(u_{11}\right)>0$. With the same scattering matrix, there is no bound state solution for OI (i.e., with $m$ sign changed). In this case the sign inversion effect is manifest, such that $m$ sign change induces appearance (disappearance) of ingap bound states. In Fig. 2(d), the spectrum in a TI-II is shown. The most significant feature is the bound states exist in arbitrarily small scattering strength, in contrast to TI-I and OI, which is consistent with the results in the previous section. We also see that the interorbital scattering $u_{I}$ has no profound affect on the bound state spectrum. In general the bound state energy migrates toward $\omega=0$ as $U$ increases, but all are somehow localized near the band edge. Nevertheless, within the parameters we are investigating, the bound states in TI-II [Fig. 2(d)] are more extended away from the band edge and these in-gap states may affect the transport property of the bulk TI.

We also include the quadratic-Dirac scatterings. The scattering matrix is given by [see Eq. (5)]

$$
\hat{v}^{\dagger}=\left[\begin{array}{cccc}
v_{+} & v_{-} & 0 & 0 \\
0 & 0 & v_{+} & v_{-}
\end{array}\right]
$$

and the eigenvalue equation is

$$
\begin{aligned}
& g_{0}^{\mathrm{q}}\left[\left(u_{11} v_{+}^{2}+u_{22} v_{-}^{2}\right) g^{+} g^{-}-v_{+}^{2} g^{+}-v_{-}^{2} g^{-}\right] \\
& \quad+\left(1-u_{11} g^{+}\right)\left(1-u_{22} g^{-}\right)=0 .
\end{aligned}
$$

Since $u_{I}$ has no profound effect on the spectrum, we set it to zero. For brevity, we shall also neglect the intra-quadratic-band scatterings. The results are shown in Fig. 3. We plot graphs of OI (a) and TI-I (b) corresponding to those in Fig. 2 with $\left|u_{I}\right|=0$. The extra $\hat{v}$ scatterings induce new bound states near the valence band edge, consistent with the results shown in Table III. It is also noted that the sign inversion effect is not manifest unless one of $v_{ \pm}$is zero such that some scattering
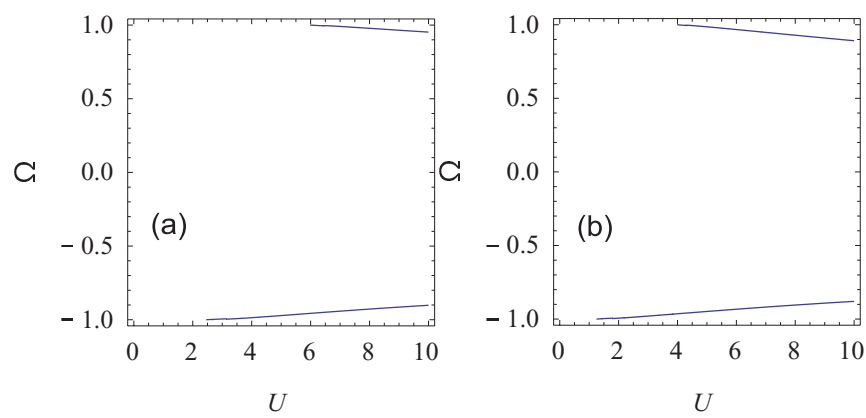

FIG. 3. (Color online) In-gap bound state spectrum with quadratic-Dirac interband scatterings. Here parameters are the same as in Figs. 2(a) and 2(b), respectively, and $\left|u_{I}\right|=0$. The interband scatterings is set as $\frac{1}{U}\left(v_{+}, v_{-}\right)=(0.6,0.5)$. 
channels are completely cut off, which is however rare in real materials.

\section{SUMMARY}

We are now in the position to discuss and summarize our results. We show that impurity scattering effect in TI with band structure parameters in certain type are enhanced compared with usual semiconductors. As a result impurity bound states can form easily in these TI, leading to enhanced bulk conductivity and reduced effective band gap. Impurity scattering effect can be reduced for TI with band parameters in suitable types (type I and III in Fig. 1 for 3D TI). Our analysis is applied to three materials $\mathrm{Bi}_{2} \mathrm{Se}_{3}, \mathrm{Bi}_{2} \mathrm{Te}_{3}$, and $\mathrm{Sb}_{2} \mathrm{Te}_{3}$, where we find that $\mathrm{Sb}_{2} \mathrm{Te}_{3}$ is the preferred material among the three as far as transport application is concerned. We note that a larger variety of TI materials exist nowadays and our analysis can be applied if their band structure is known.

We also point out another interesting sign-inversion effect associated with band inversion in TI-OI transition. The effect is most pronounced in $2 \mathrm{D}$ TI with $2 m B<1$. Experimentally, the TI-OI transition can be controlled by gate voltage in
InAs/GaSb quantum well system and the transport measurement can be carried out at various chemical potentials inside the band gap. ${ }^{23,24}$ The band inversion effect can be observed through a change in distribution of impurity bound state energies when the system changes from TI to OI state. The effect is strongest if the impurities coupled preferably to one of the atomic orbitals.

In conclusion, we study the effect of impurity scattering in TI in this paper. Our work is complimentary to previous works $^{14-16}$ that consider impurity bound state forming from continuous deformation of edge modes. We find that impurityinduced bound state formation depends strongly on the band structure of the TI and impurity scattering can be suppressed in TI with band structures in the correct types. Our result provides a guidance for TI material engineering which is useful for search of applicable TI.

\section{ACKNOWLEDGMENTS}

We acknowledge helpful discussion with S. Q. Shen. This work is supported by HKRGC through Grant CRF09/HKUST03.
${ }^{1}$ M. Z. Hasan and C. L. Kane, Rev. Mod. Phys. 82, 3045 (2010).

${ }^{2}$ X.-L. Qi and S.-C. Zhang, Rev. Mod. Phys. 83, 1057 (2011).

${ }^{3}$ J. C. Y. Teo and C. L. Kane, Phys. Rev. B 82, 115120 (2010).

${ }^{4}$ J. E. Moore, Nature (London) 464, 194 (2010).

${ }^{5}$ J. G. Checkelsky, Y. S. Hor, M.-H. Liu, D.-X. Qu, R. J. Cava, and N. P. Ong, Phys. Rev. Lett. 103, 246601 (2009).

${ }^{6}$ D.-X. Qu, Y. S. Hor, J. Xiong, R. J. Cava, and N. P. Ong, Science 329, 821 (2010).

${ }^{7}$ J. G. Analytis, R. D. McDonald, S. C. Riggs, J.-H. Chu, G. S. Boebinger, and I. R. Fisher, Nat. Phys. 6, 960 (2010).

${ }^{8}$ J. G. Analytis, J.-H. Chu, Y. Chen, F. Corredor, R. D. McDonald, Z. X. Shen, and I. R. Fisher, Phys. Rev. B 81, 205407 (2010).

${ }^{9}$ K. Eto, Z. Ren, A. A. Taskin, K. Segawa, and Y. Ando, Phys. Rev. B 81, 195309 (2010).

${ }^{10}$ N. P. Butch, K. Kirshenbaum, P. Syers, A. B. Sushkov, G. S. Jenkins, H. D. Drew, and J. Paglione, Phys. Rev. B 81, 241301 (2010).

${ }^{11}$ Z. Ren, A. A. Taskin, S. Sasaki, K. Segawa, and Y. Ando, Phys. Rev. B 82, 241306 (2010).

${ }^{12}$ Z. Ren, A. A. Taskin, S. Sasaki, K. Segawa, and Y. Ando, Phys. Rev. B 84, 165311 (2011).
${ }^{13}$ J. Xiong, A. C. Petersen, D. Qu, Y. S. Hor, R. J. Cava, and N. P. Ong, Physica E (2011), doi: 10.1016/j.physe.2011.09.011.

${ }^{14}$ W.-Y. Shan, J. Lu, H.-Z. Lu, and S.-Q. Shen, Phys. Rev. B 84, 035307 (2011)

${ }^{15}$ S.-Q. Shen, W.-Y. Shan, and H.-Z. Lu, SPIN 1, 33 (2011).

${ }^{16}$ J. Lu, W.-Y. Shan, H.-Z. Lu, and S.-Q. Shen, New J. Phys. 13, 103016 (2011).

${ }^{17}$ L. Fu and C. L. Kane, Phys. Rev. B 76, 045302 (2007).

${ }^{18}$ H. Zhang, C.-X. Liu, X.-L. Qi, X. Dai, Z. Fang, and S.-C. Zhang, Nat. Phys. 5, 438 (2009).

${ }^{19}$ V. V. Cheianov and V. I. Fal'ko, Phys. Rev. Lett. 97, 226801 (2006).

${ }^{20}$ A. V. Balatsky, I. Vekhter, and J.-X. Zhu, Rev. Mod. Phys. 78, 373 (2006).

${ }^{21}$ T. K. Ng and Y. Avishai, Phys. Rev. B 80, 104504 (2009).

${ }^{22}$ C.-X. Liu, X.-L. Qi, H. J. Zhang, X. Dai, Z. Fang, and S.-C. Zhang, Phys. Rev. B 82, 045122 (2010).

${ }^{23}$ I. Knez, R.-R. Du, and G. Sullivan, Phys. Rev. Lett. 107, 136603 (2011).

${ }^{24}$ I. Knez, R.-R. Du, and G. Sullivan, e-print arXiv:1106.5819. 